\title{
El maltrato entre escolares y otras conductas-problema para la convivencia
}

\author{
Santiago Ramírez Fernández
}

$\mathrm{y}$

Fernando Justicia Justicia

Departamento de Psicología Evolutiva y de la Educación Universidad de Granada

\section{España}

sramirez@ugr.es

fjusticia@ugr.es 


\section{Resumen}

Introducción: Hoy en día, el maltrato entre escolares por abuso de poder es conceptualizado como un fenómeno complejo y multicausal para cuyo estudio se hace necesario adoptar una perspectiva más ecológico-social y menos individualista, donde el grupo de iguales se convierta en una unidad de análisis indiscutible. Con este trabajo nos hemos planteado como objetivo relacionar el mayor o menor número de las conductas-problemas informadas por el profesor con los distintos grados de implicación de los protagonistas de malos tratos, examinando también las diferencias por género.

Método: Hemos trabajado con 24 unidades completas de dos centros públicos de la ciudad autónoma de Ceuta en los niveles que van desde $3^{\circ}$ de Educación Primaria a $2^{\circ}$ curso de la Educación Secundaria Obligatoria. Aplicamos, en cada grupo-clase, dos cuestionarios: uno para evaluar la incidencia del maltrato e identificar a los distintos protagonistas y, otro, con el propósito de conocer, desde la perspectiva del profesor, con qué frecuencia ejercitan sus alumno distintas conductas-problemas para la convivencias. Efectuamos análisis de varianza (univariante y multivariante) con los que pudimos comparar y estudiar las posibles diferencias existentes entre los distintos niveles de las variables independientes.

Resultados: Los resultados obtenidos muestran que la variable "Tipología de los implicados en maltrato" es muy discriminativa con respecto a los problemas de convivencia. Los implicados de forma más directa (víctimas, intimidadores e intimidadores-victimizados) participan en mayor medida que espectadores implicados y espectadores puros de otros problemas que deterioran la convivencia. Sin embargo, los espectadores implicados obtienen unas puntuaciones en cada uno de los factores de convivencia estudiados que varían de forma similar a como lo hacen las víctimas, pero con menores promedios. Con relación al género, las víctimas mujeres presentan diferencias significativas con respecto a las víctimas hombres en conductas como desinterés académico y habilidades de comunicación con el grupo.

Discusión: Aparece evidencia empírica que apoya la hipótesis que relaciona la frecuencia de conductas problemáticas para la convivencia y el tipo de implicación de los alumnos en situaciones de maltrato. La evolución equivalente que siguen los espectadores implicados con relación a las víctimas debe llevarnos a que la investigación tome cada vez más en serio los comportamientos violentos de baja o moderada frecuencia.

Palabras clave: maltrato escolar, convivencia, víctima, intimidador, conflictividad escolar. 


\section{Introducción}

No podemos sustraernos de vivir-con otros. La vida junto a otras personas es inevitable en cualquier sociedad. Comenzamos "con-viviendo" con aquellos que constituyen nuestro núcleo familiar y conforme crecemos nos vamos incorporando a nuevos grupos que despliegan su actividad en escenarios diferentes. Uno de estos espacios del que no podemos escapar, en las sociedades avanzadas, es la escuela. El paso obligado por esta institución y el periodo, cada vez más largo, de permanencia hacen que nos planteemos este lugar como un sitio idóneo desde el que enseñar a convivir a nuestros jóvenes. No obstante, el centro escolar, a pesar de gozar de estas ventajas, no es el contexto más fácil para hacerlo. La progresiva complejidad que está adquiriendo la escuela hace de este entorno un lugar complicado para que los que acuden a ella mantengan una buena convivencia. Se dan muchos elementos, muchas variables, que hacen que este territorio compartido sea propicio a la aparición de conflictos que pueden devenir en violencia o en impedir que la escuela cumpla eficazmente con las funciones que se le han encomendado. Pensemos si no en las siguientes cuestiones (Ianni y Pérez, 1998):

- Cada vez observamos una mayor distancia generacional entre los adultos y jóvenes que asisten a la escuela. Esto se traduce en la existencia de unos intereses y unos valores muy distintos entre ellos.

- $\quad$ La escuela está asumiendo en los últimos años una diversidad creciente de funciones. Estamos asistiendo a un desentendimiento de la familia y otras instituciones sociales en el desempeño de sus funciones y esta dejación de tareas está siendo asumida por la escuela, con lo que la estamos sobrecargando.

- $\quad$ Muchos de los cambios que se producen en la sociedad no atraviesan las paredes de la escuela. Se detienen a sus puertas. En numerosas ocasiones, se tiene la impresión de estar viviendo de espaldas a la realidad en la que estamos insertos. Nos cuesta, desde la escuela, conectar con lo que se está viviendo fuera.

- $\quad$ El contexto escolar es cada vez más diverso, la realidad multicultural de nuestras sociedades hace que dentro de las escuelas vivamos experiencias muy diferentes.

- $\quad$ La escuela está soportando, últimamente, una presión importante de los medios de comunicación a los que sólo parece interesarles los problemas y los fallos que tiene esta institución. 
- Del mismo modo, el profesor no está contando con el apoyo y el reconocimiento a su labor, por parte de la sociedad, lo que hace menos gratificante su trabajo y paraliza muchas actuaciones innovadoras.

Estas cuestiones, como podemos imaginar, pueden dificultar las relaciones entre los distintos protagonistas que interactúan en la escuela y desembocar en una mala convivencia. Hay otros aspectos, inherentes a la propia naturaleza de la escuela, que tampoco contribuyen, precisamente, a la construcción de una buena convivencia. Estamos hablando, por ejemplo, de la obligatoriedad de la escuela que reúne niños hasta los 16 años en un recinto en el que se ven obligados a convivir con otros niños y otros adultos, en principio desconocidos para ellos. Han de compartir unos espacios y un tiempo en una estructura organizativa diseñada y controlada por adultos. Tienen que adaptarse, para ello, a un reglamento con medidas disciplinarias que han diseñado otros y deben aceptar el poder que emana de una autoridad impuesta por el sistema y que ellos no han elegido. Además, han de iniciar juntos un proceso de aprendizaje no exento de problemas: los profesores tienen que enseñar unos contenidos que muchas veces no responde a las necesidades e intereses de los niños; se empeñan en enseñarlos de una manera y los chicos quieren aprenderlos de otra; la dinámica organizativa y la distribución de roles que se imponen en este proceso no suele corresponderse con las ideas que sobre ello tienen los alumnos, etc. (Pereira, 2002). No obstante, pese a sus carencias, la escuela sigue siendo la institución social que aglutina a un importante número de niños, adolescentes y jóvenes. Por ello, hay que esforzarse en conseguir que el periodo en el que los niños permanezcan en el centro escolar sea un tiempo de crecimiento, de desarrollo personal y social. Que la escuela se convierta en un lugar por el que "circule la palabra y no los silencios, el diálogo y no la sumisión y el acatamiento, el análisis y la reflexión sobre las acciones impulsivas y las actuaciones violentas." (Ianni, 2003, p. 1).

Como podemos ver, el centro escolar, como escenario institucional de convivencia, no escapa a los problemas que ésta conlleva y por consiguiente, ha sido objeto de bastantes titulares y estudios durante los últimos años. El foco de atención de estos trabajos sobre convivencia en la escuela se ha centrado, en gran medida, en la temática de la violencia escolar. Esto ha ocasionado un aumento en la producción teórica y práctica sobre este fenómeno. Los análisis, las reflexiones y las intervenciones para mejorar la convivencia escolar han girado, por tanto, en torno a la violencia generada en los establecimientos educativos.

Sin desdeñar la importancia y el enorme peso que tiene el tema de la violencia escolar, hemos creído que puede resultar conveniente destacar otros aspectos que "nos ayuden a examinar la convivencia en los centros desde una perspectiva un poco más amplia, y que apunte a mostrar la 
realidad con toda su complejidad" (Morollón, 2001, p.42). Por ello, un tratamiento más extenso a la hora de afrontar la conflictividad escolar requiere de un análisis e identificación de las distintas conductas-problemas que perturban la convivencia. Son muchos los intentos de categorizar este cúmulo de conductas que ocurren en los centros escolares. Con mayor o menor grado de especificidad a la hora de hacerlo, la mayoría de las clasificaciones giran en torno a una serie de grupos muy similares. Así, por ejemplo, Ortega (2002) distingue 5 categorias de conductas desadaptadas en el contexto escolar: vandalismo o violencia contra los objetos, los bienes, etc; disruptividad o violencia contra las finalidades educativas; indisciplina o violencia contra las convenciones con las que se gobierna la actividad escolar; maltrato personal y violencia interpersonal con resultados penales. Por su parte Calvo (2003) habla de conductas de rechazo al aprendizaje, conductas de trato inadecuado, conductas disruptivas y conductas agresivas.

Nosotros hemos seguido las 5 dimensiones racionales de las que partió Peralta (2004) para elaborar el Cuestionario sobre Problemas de Convivencia Escolar y que se asemejan mucho a las elaboradas por otros autores como los citados anteriormente:

-Desinterés académico: Las conductas de desinterés académico o de rechazo al aprendizaje hacen referencia a aquellos comportamientos del alumno, normalmente observados en clase, que van encaminados a no realizar las tareas académicas propuestas por el profesor.

- Conducta disruptiva: La disrupción es "la música de fondo de la mayoría de nuestras aulas" (Torrego y Moreno, 2003, p.129). Cuando hablamos de disrupción estamos haciendo referencia a un conjunto de comportamientos que deterioran o interrumpen el proceso de enseñanza y aprendizaje del aula.

- Conductas agresivas: Son comportamientos cometidos para causar intencionalmente daño a otra persona.

- Conductas indisciplinadas: Son conductas que suponen un desacato a las normas de organización y funcionamiento presentes en un centro. No parecen tener la intención, en este caso, de molestar con ellas a sus compañeros ni a sus profesores.

- Conductas antisociales: Con este tipo de comportamientos se intenta atentar contra la integridad física o psíquica de los demás o contra las pertenencias individuales o comunitarias.

Desde esta realidad nuestro objetivo con este estudio es el de relacionar el mayor o menor número de las conductas-problemas informadas por el profesor con los distintos grados de implicación de los protagonistas de malos tratos por abuso de poder. Esperamos encontrar diferencias significativas en las puntuaciones obtenidas en las conductas problemáticas para la convivencia por los 
diversos tipos de implicados en malos tratos. Del mismo modo queremos analizar la influencia que variables como el sexo tienen en la aparición y caracterizacion de los problemas de convivencia. Esperamos, por tanto, que en el estudio que realicemos sobre las interacciones entre la variable sexo y la tipología de implicados con respecto a las puntuaciones obtenidas en los factores de convivencia aparezcan diferencias significativas.

\section{Método}

\section{Participantes}

El número de sujetos que ha formado parte de la muestra ha sido de 587 , lo que supone el 13,6\% de participantes con relación a la población total objeto de estudio. Los alumnos y alumnas seleccionados proceden de dos centros públicos de Educación Primaria de la Ciudad Autónoma de Ceuta. Uno de ellos recibe alumnado de toda la zona centro de la ciudad y del "Campo Interior" (zona adyacente a aquella) y el otro está ubicado en el "Campo Exterior", una zona suburbana en la que vive mayoritariamente población musulmana. Ambos colegios en el momento de incorporarse a esta investigación eran Centros de Primaria en los que se seguía impartiendo el primer ciclo de la Educación Secundaria Obligatoria.

De los 587 sujetos que constituyen la muestra, 318 son varones $(54,2 \%)$ y 269 son mujeres $(45,8 \%)$ y se mueven en un rango de edad que va desde los ocho a los quince años. Proporciones éstas muy semejantes a las que aparecen en la población de origen. Los niveles educativos de donde hemos obtenido a los alumnos/as participantes han sido $3^{\circ}, 4^{\circ}, 5^{\circ}$ y $6^{\circ}$ de Primaria y $1^{\circ}$ y $2^{\circ}$ de Educación Secundaria Obligatoria. En ambos centros pudimos contar con dos líneas completas de escolaridad (un grupo A y un grupo B) para cada uno de los niveles educativos antes citados (24 unidades en total), por lo que no existen apenas diferencias en la proporción de sujetos que están representados en ellos.

\section{Instrumentos}

Para la recogida de datos hemos utilizado dos cuestionarios ya elaborados y contrastados por diferentes autores y que nosotros nos hemos encargado de adaptar a la realidad en la que vamos a trabajar. Estos son:

El cuestionario sobre problemas de convivencia escolar en Secundaria (CPCE) (Peralta, 2004). 
- $\quad$ El cuestionario sobre maltrato entre escolares (Olweus, 1993, modificado por Ortega y Mora-Merchán)

El primer cuestionario recoge información, desde el punto de vista del profesor, de las diferentes conductas-problemas que deterioran la convivencia en el centro. Tras las distintas modificaciones realizadas al cuestionario de partida (eliminación de ítems, concreción y reformulación de items, inclusión de nuevos ítems, etc.) la evaluación de las citadas conductas se hizo a través de 96 items, en una escala de 4 intervalos, que debían cumplimentar los tutores de todos los cursos para cada uno de sus alumnos. Respetamos las 5 dimensiones racionales que utilizó el autor para la confección del cuestionario y que ya hemos definido anteriormente (conducta indisciplinada, conducta de desinterés académico, conducta antisocial, conducta de maltrato entre compañeros, conducta disruptiva).

El segundo de los instrumentos utilizados fue adaptado para las unidades de Educación Primaria, teniendo en cuenta las dificultades de comprensión de la lengua española que presentan los alumnos musulmanes escolarizados en estos niveles y los problemas de interpretación conceptual que tienen los niños escolarizados en los niveles más bajos. Con este cuestionario se pretende que los distintos protagonistas que participan en las situaciones de maltrato entre compañeros se autoidentifiquen señalando el papel que juegan y las opiniones que le merecen algunas cuestiones relacionadas con este problema.

\section{Procedimiento}

La aplicación del cuestionario de malos tratos se realizó en las aulas en situación de grupo-clase, en una sesión ordinaria del horario de mañana. La cumplimentación fue voluntaria, anónima y, durante el tiempo que duró, los tutores no estuvieron presentes. Comenzábamos explicando a los alumnos qué pretendiamos con el trabajo, en qué aspectos solicitábamos su colaboración, de qué forma íbamos a garantizar la confidencialidad de sus respuestas y lo importante que era para nosotros que nos respondieran con total sinceridad. Seguidamente, clarificábamos determinados conceptos que considerábamos debían compartir todos antes de contestar, como por ejemplo, el concepto y las diferentes modalidades de maltrato que debían tener en cuenta. Esto lo íbamos explicando con ayuda de las ilustraciones existente en el cuestionario de Primaria. Por último, informábamos de los pasos y las acciones que tenían que realizar para cumplimentar correctamente el cuestionario. 
Del mismo modo, mantuvimos varias reuniones con el profesorado en las que se informó de las distintas dimensiones sobre las que queríamos recoger información, de la importancia de su colaboración y sinceridad y se proporcionaron algunas instrucciones para la cumplimentación de los mismos.

Ambos cuestionarios fueron pasados durante el mismo periodo de tiempo para captar una misma realidad desde puntos de vistas diferentes. Tardamos, aproximadamente, dos meses en recoger toda la información.

\section{Análisis Estadístico}

Hemos hecho un uso de la estadística en los tres niveles de aplicación: nivel descriptivo, nivel inferencial y multivariante, de tal manera que, con ellos, hemos podido profundizar en estos análisis aprovechando al máximo la información que contenían los datos proporcionados por la muestra estudiada. Estos resultados se presentan de forma completa en la tesis doctoral realizada por uno de los autores y dirigida por el otro (Ramírez, 2006). En el caso concreto del estudio que aquí estamos exponiendo hemos empleado el análisis de varianza de una clasificación por rango de Kruskal-Wallis que representa una alternativa útil para $\mathrm{K}$ muestras independientes y que sirve para decidir si aquellas muestras son de poblaciones diferentes. Kruskal y Wallis (1952) ya la presentaron como una de las pruebas más eficientes entre las no paramétricas y nosotros las hemos utilizado por sus características en un primer acercamiento al estudio de las diferencias en el ámbito de la convivencia, siempre que las variables permitían la adaptación a este tipo de prueba.

Siguiendo a Tejedor (1984) las condiciones paramétricas para aplicar el análisis de varianza pueden flexibilizarse siempre y cuando "no sean muy notorios los incumplimientos de ellas, especialmente el de la no normalidad" (p.277). Por ello, pueden aplicarse a datos medidos al menos en rango que puedan reflejar continuidad, siempre que las muestras sean amplias (más de 300). Este análisis proporciona unos resultados amplios y, además, aporta información sobre lo que denominamos comparaciones a posteriores (post-hoc), localizando exactamente entre qué dimensiones de la variable se encuentran las diferencias. Por esta razón la hemos utilizado y para las pruebas a posteriori, hemos elegido la denominada como "diferencias mínimas significativas" (DSM), que es la menos conservadora (entendiendo por conservadora aceptar una diferencia que no lo es) o sea la máxima potencia de la prueba (Etxeberría, Joaristi y Lizasoain, 1990). 
Para el estudio de la interacción entre variables hemos empleado el análisis de multivarianza (MANOVA) que nos ha permitido en este trabajo profundizar en el nivel inferencial destacando la acción conjunta de las variables entre sí. Para una interpretación todavía más ajustada de los efectos de una interacción entre dos variables independientes hemos utilizado la ayuda que nos proporcionan los gráficos de perfil.

\section{Resultados}

\section{Cuántos son los implicados en las situaciones de maltrato y que roles desempeñan}

Una primera aproximación global al problema nos permite observar que el nivel de conflictividad en el terreno de las relaciones interpersonales entre los alumnos es bastante considerable. Estamos hablando de un 57,6\% de escolares que afirman haber sufrido, al menos en una ocasión, algún tipo de agresión física, verbal o psicológica durante el primer trimestre del curso y de un $38,3 \%$ de sujetos que reconocen haber ejercido algún maltrato sobre otro compañero durante el mismo periodo de tiempo. Es decir, que uno de cada dos estudiantes ha sido agredido, en alguna ocasión, por los compañeros del centro y uno de cada tres ha atacado, en algún momento, a otros chicos y chicas en el entorno escolar.

Como era previsible, no todos los afectados han sido objeto de malos tratos con igual frecuencia y duración. El grupo más preocupante lo conforman 43 alumnos (7,4\% del total de la muestra) que denuncian ser maltratados "casi todos los días" (maltrato severo) por sus compañeros del centro. Estos podrían ser catalogados como víctimas "puras”, sujetos que están padeciendo verdaderas situaciones de intimidación. Ha sido, de hecho, este criterio restrictivo el que hemos utilizado para elaborar la tipología de alumnos implicados en situaciones de maltrato propiamente dicha. La razón que nos ha llevado a adoptar este criterio extremo se encuentra en las múltiples investigaciones (Almeida, 1999; Benítez, 2003; MoraMerchán, 2000; Olweus, 1993; Salmivalli, Lagerspetz, Bjoerkqvist, Oesterman y Kaukianinen, 1996; Wihney y Smith, 1993) realizadas con el fin de valorar el predominio o incidencia del maltrato en los centros escolares. En ellas, se recoge la necesidad de tomar criterios restrictivos si se quiere identificar, con ciertas garantías, a víctimas y agresores. Solberg y Olweus (2003) reafirman lo dicho en una reciente investigación.

Del mismo modo, los agresores actúan con intensidades diferentes a la hora de ejercer maltrato sobre sus compañeros.Quiénes hacen de la intimidación una conducta habitual en 
ellos (casi diaria) suponen el 4,1\% del total de sujetos estudiados. Siguiendo el mismo criterio que para las víctimas, éstos últimos son los que hemos considerado verdaderos intimidadores.

Long y Pellegrini (2003) y Rodkin y Hodges (2003) consideran la intimidación como un fenómeno de grupo y sugieren, por tanto, que hay que atender a los diversos roles que los escolares pueden desempeñar (agresor, víctima, agresor-victimizado, espectador,... Salmivalli et al., 1996; Swearer, Song, Cary, Eagle y Mickelson, 2001) e ir abandonando la primera categoría dicotómica en la que sólo eran clasificados los protagonistas como intimidadores o como víctimas.

Siendo coherentes con estas ideas, hemos establecido un sistema de clasificación que recogiese cada uno de los papeles que los estudiantes pueden desempeñar en las situaciones de malos tratos severos.

Para ello, hemos tenido en cuenta las preguntas 2 y 6 del cuestionario de Primaria sobre malos tratos (2P y 6P) y las preguntas 3 y 16 del cuestionario de Secundaria (3S y 16S). Con las preguntas $2 \mathrm{P}$ y $3 \mathrm{~S}$ solicitamos a los escolares que nos informen del número de veces que han sido objeto de malos tratos por parte de sus compañeros. Del mismo modo, con las preguntas $6 \mathrm{P}$ y $16 \mathrm{~S}$, se requería también la frecuencia pero del número de ocasiones en que habían ejercido acciones intimidatorias hacia sus compañeros.

El procedimiento utilizado para clasificar a los alumnos participantes en los distintos roles que pueden darse en las situaciones de intimidación, es el mismo que el seguido por Mora-Merchán (2000), consistente en cruzar, en una tabla de doble entrada, toda la información, ya codificada, proveniente de las dos preguntas anteriormente citadas. En un lado de la tabla estaría la pregunta referida a las experiencias de victimización con todas sus opciones de respuesta y en el otro la pregunta utilizada para recoger información sobre las vivencias de los sujetos como agresores, igualmente, con todas sus respuestas. Del cruce de ambas obtenemos las distintas categorías posibles de participación en situaciones de malos tratos.

Así los que comunican haber padecido, con una frecuencia casi diaria, agresiones de sus compañeros son los que hemos catalogados como víctimas, los que manifiestan con la misma frecuencia haber maltratado a sus compañeros serían los intimidadores y aquellos que manifiestan haber agredido y recibido agresiones al mismo tiempo, con igual frecuencia, serían los intimidadores-victimizados. Quedaría un número importante de sujetos que no están implicados en las situaciones de malos tratos o lo están de forma poco severa y que hemos denominado espectadores. Estos a su vez, los hemos subdivididos en Espectadores no Impli- 
cados (aquellos que no han participado en situaciones de maltrato) y Espectadores Implicados (aquellos que, en alguna ocasión han participado desempeñando el papel de víctimas o agresores). En la tabla siguiente puede observarse el porcentaje de sujetos que forma parte de cada tipología.

Tabla 1. Tipología de implicados. Porcentajes

\begin{tabular}{|c|c|c|c|c|c|c|}
\hline & \multicolumn{5}{|c|}{ ¿Cuántas veces has maltratado a tus compañeros? } \\
\hline & & Nunca & $\begin{array}{l}\text { Alguna } \\
\text { vez (1 } \\
\text { ó 2) }\end{array}$ & $\begin{array}{l}\text { Pocas } \\
\text { veces } \\
(\text { Entre } \\
3 \text { y 6) }\end{array}$ & $\begin{array}{l}\text { Bastantes } \\
\text { veces } \\
\text { (Más de } \\
6 \text { ) }\end{array}$ & $\begin{array}{c}\text { Muchas veces } \\
\text { (Casi todos los } \\
\text { días) }\end{array}$ \\
\hline \multirow{5}{*}{$\begin{array}{l}\text { ¿Cuántas } \\
\text { veces has } \\
\text { recibido } \\
\text { maltrato de } \\
\quad \text { tus } \\
\text { compañeros? }\end{array}$} & Nunca & \multirow{4}{*}{\multicolumn{4}{|c|}{$\begin{array}{cc} & \begin{array}{l}\text { No implicado } \\
(33,3 \%)\end{array} \\
\begin{array}{cc}\text { Espectadores } \\
(89,5 \%)\end{array} & \\
& \text { Implicados } \\
& (\mathbf{5 6 , 2 \% )}\end{array}$}} & \multirow{4}{*}{$\begin{array}{c}\text { Intimidadores } \\
\text { o Agresores } \\
(\mathbf{3}, \mathbf{1 \%} \%)\end{array}$} \\
\hline & $\begin{array}{c}\text { Alguna } \\
\text { vez ( } 1 \text { ó } \\
2) \\
\end{array}$ & & & & & \\
\hline & $\begin{array}{c}\text { Pocas } \\
\text { veces } \\
\text { (Entre } 3 \\
\text { y 6) } \\
\end{array}$ & & & & & \\
\hline & $\begin{array}{c}\text { Bastantes } \\
\text { veces } \\
\text { (Más de } \\
6 \text { ) } \\
\end{array}$ & & & & & \\
\hline & $\begin{array}{l}\text { Muchas } \\
\text { veces } \\
\text { (Casi } \\
\text { todos los } \\
\text { días) }\end{array}$ & \multicolumn{4}{|c|}{$\begin{array}{c}\text { Víctimas } \\
(6,4 \%)\end{array}$} & $\begin{array}{c}\text { Intimidadores } \\
\text { o Agresores/ } \\
\text { Victimizados } \\
(1 \%)\end{array}$ \\
\hline
\end{tabular}

Si sumamos el porcentaje de víctimas existente, con el de agresores y el de intimidadores victimizados obtenemos que, en nuestra investigación, el número de implicados directamente y de forma severa en acciones intimidatorias alcanza el 10,5\% de los sujetos estudiados.

\section{El maltrato entre escolares en el contexto de los problemas de convivencia}

En este bloque de resultados queremos destacar el tipo de relaciones que hemos encontrado entre la tipología de implicados en situaciones de maltrato y las puntuaciones obtenidas en el cuestionario sobre problemas de convivencia en los centros. Estas puntuaciones han sido calculadas para cada uno de los 7 factores que han aparecido en el análisis factorial realizado al respecto $(\mathrm{F} 1=$ Conductas agresivas hacia los compañeros; $\mathrm{F} 2=$ Desinterés académico; $\mathrm{F} 3=$ Conductas disruptivas; F4= Conductas exhibicionistas y groseras; F5= Falta de habilidades 
para la comunicación con el grupo; F6= Conductas agresivas hacia profesores; F7= Conductas delictivas.

Interdependencia entre los Tipos de implicados en maltrato y el CPCEA.

Los resultados obtenidos en la prueba no paramétrica de Kruskal-Wallis, confirman que la variable "Tipología de los implicados en maltrato" es muy discriminativa con respecto a los problemas de convivencia. Todos los factores extraídos del Cuestionario de Problemas de Convivencia Escolar Adaptado (CPCEA), así como la puntuación total del mismo, muestran efectos estadísticamente significativos para la Variable Independiente antes citada. Estos datos los mostramos en la siguiente tabla.

Tabla 2. Efectos aparecidos para la variable independiente Tipología de Implicados respecto a las puntuaciones del CPCEA

\begin{tabular}{|c|c|c|c|c|c|c|c|c|}
\hline & \multicolumn{3}{|c|}{ Tipología de implicados } & \multicolumn{5}{|c|}{ Kruskal-Wallis } \\
\hline & $\begin{array}{l}\text { Víctimas } \\
\text { N=37 } \\
\text { M(dt.) }\end{array}$ & $\begin{array}{c}\text { Agresores } \\
\mathrm{N}=18 \\
\mathrm{M}(\mathrm{dt} .)\end{array}$ & $\begin{array}{c}\text { Agres-vict } \\
\mathrm{N}=6 \\
\mathrm{M}(\mathrm{dt} .)\end{array}$ & $\begin{array}{l}\text { Espect } \\
\text { Implica } \\
\mathrm{N}=326 \\
\mathrm{M}(\mathrm{dt} .)\end{array}$ & $\begin{array}{c}\text { Espect } \\
\text { Puros } \\
\mathrm{N}=192 \\
\mathrm{M}(\mathrm{dt} .)\end{array}$ & $\mathrm{X}^{2}$ & Gl & $\mathrm{p}<$ \\
\hline $\mathrm{F} 1$ & $1,58(.75)$ & $1,59(.56)$ & $1,65(.68)$ & $1,42(.56)$ & $1,21(.38)$ & 35,844 & 4 & .000 \\
\hline $\mathrm{F} 2$ & $1,99(.91)$ & $1,71(.69)$ & $2,61(1.08)$ & $1,64(.64)$ & $1,49(.60)$ & 23,582 & 4 & .000 \\
\hline F3 & $1,80(.77)$ & $1,88(.63)$ & $2,13(.82)$ & $1,57(.66)$ & $1,28(.42)$ & 52,459 & 4 & .000 \\
\hline F4 & $1,21(.44)$ & $1,12(.26)$ & $1,23(.40)$ & $1,09(.28)$ & $1,04(.20)$ & 20,631 & 4 & .000 \\
\hline F5 & $1,75(.91)$ & $1,57(.63)$ & $2,13(.86)$ & $1,34(.54)$ & $1,16(.37)$ & 39,995 & 4 & .000 \\
\hline F6 & $1,32(.60)$ & $1,52(.65)$ & $1,50(.63)$ & $1,21(.49)$ & $1,04(.19)$ & 51,212 & 4 & .000 \\
\hline F7 & $1,08(.17)$ & $1,02(.08)$ & $1(.00)$ & $1.04(.21)$ & $1,007(.04)$ & 21,034 & 4 & .000 \\
\hline $\mathrm{T}$ & $1,53(.58)$ & $1,49(.37)$ & $1,75(.59)$ & $1,33(.38)$ & $1,18(.23)$ & 45,915 & 4 & .000 \\
\hline
\end{tabular}

En los análisis de varianza, la variable independiente Tipología de los implicados en maltrato también ha mostrado efectos estadísticamente significativos en todos los factores y en la puntuación total del CPCEA. 
Tabla 3. ANOVA. Efectos aparecidos para la variable independiente Tipología de Implicados respecto a las puntuaciones del CPCEA

\begin{tabular}{|c|c|c|c|c|c|c|c|}
\hline & $\begin{array}{c}\text { Víctimas } \\
\mathrm{N}=37 \\
\mathrm{M}(\mathrm{dt} .)\end{array}$ & $\begin{array}{c}\text { Agresores } \\
\mathrm{N}=18 \\
\mathrm{M}(\mathrm{dt} .)\end{array}$ & $\begin{array}{c}\text { Agres-vict } \\
\quad N=6 \\
M(d t .)\end{array}$ & $\begin{array}{c}\text { Espect } \\
\text { Implica } \\
\mathrm{N}=326 \\
\mathrm{M}(\mathrm{dt} .)\end{array}$ & $\begin{array}{c}\text { Espect } \\
\text { Puros } \\
\mathrm{N}=192 \\
\mathrm{M} \text { (dt.) }\end{array}$ & $\mathrm{F}$ & Post hoc \\
\hline F1 & $1,58(.75)$ & $1,59(.56)$ & 1,65(.68) & $1,42(.56)$ & $1,21(.38)$ & $7,685^{\star \star \star \star}$ & $\begin{array}{l}1>5^{\star * \star *} \\
2>5^{\star *} \\
3>5^{*} \\
4>5^{\star * * *} \\
1>4^{\star *}\end{array}$ \\
\hline F2 & $1,99(.91)$ & $1,71(.69)$ & $2,61(1.08)$ & $1,64(.64)$ & $1,49(.60)$ & $8,230^{\star * * *}$ & $\begin{array}{l}1>5^{\star \star \star *} \\
3>1^{*} \\
3>2^{* *} \\
3>4^{\star \star \star *} \\
3>5^{\star \star \star *} \\
4>5^{\star *} \\
1>4^{*}\end{array}$ \\
\hline F3 & $1,80(.77)$ & $1,88(.63)$ & $2,13(.82)$ & $1,57(.66)$ & $1,28(.42)$ & $13,445^{\star * * *}$ & $\begin{array}{l}1>5^{\star \star \star \star} \\
2>4^{*} \\
2>5^{\star \star \star \star} \\
3>4^{*} \\
3>5^{\star \star *} \\
4>5^{\star \star \star *} \\
1>4^{*}\end{array}$ \\
\hline F4 & $1,21(.44)$ & $1,12(.26)$ & $1,23(.40)$ & $1,09(.28)$ & $1,04(.20)$ & $3,631^{* *}$ & $\begin{array}{l}1>5^{\star \star *} \\
4>5^{\star} \\
1>4^{\star * \star *}\end{array}$ \\
\hline F5 & $1,75(.91)$ & $1,57(.63)$ & $2,13(.86)$ & $1,34(.54)$ & $1,16(.37)$ & $14,470^{\star * * *}$ & $\begin{array}{l}1>5^{\star \star \star \star *} \\
2>5^{\star \star} \\
3>2^{\star} \\
3>4^{\star \star \star \star} \\
3>5^{\star \star \star *} \\
4>5^{\star \star \star \star}\end{array}$ \\
\hline F6 & $1,32(.60)$ & $1,52(.65)$ & $1,50(.63)$ & $1,21(.49)$ & $1,04(.19)$ & $9,755^{\star \star \star *}$ & $\begin{array}{l}1>5^{\star * * *} \\
2>4^{\star *} \\
2>5^{\star * * *} \\
3>5^{\star} \\
4>5^{\star * * *}\end{array}$ \\
\hline F7 & $1,08(.17)$ & $1,02(.08)$ & $1(.00)$ & $1.04(.21)$ & $1,007(.04)$ & $2,391^{*}$ & $\begin{array}{l}1>5^{\star *} \\
4>5^{\star}\end{array}$ \\
\hline $\mathrm{T}$ & $1,53(.58)$ & $1,49(.37)$ & $1,75(.59)$ & $1,33(.38)$ & $1,18(.23)$ & $13,476^{\star \star \star *}$ & $\begin{array}{l}1>4^{\star \star *} \\
1>5^{\star \star \star \star} \\
2>5^{\star \star *} \\
3>4^{\star \star} \\
3>5^{\star \star \star \star} \\
4>5^{\star \star \star \star}\end{array}$ \\
\hline
\end{tabular}

Al comparar, entre sí, los distintos grupos de implicados en malos tratos con relación a las distintas puntuaciones del CPCEA, obtuvimos las siguientes diferencias significativas entre los mismos.

Factor 1 (Conductas agresivas hacia alumnos): Como era de esperar, los alumnos autoidentificados como víctimas, agresores y agresores victimizados puntúan en este factor más que los espectadores implicados ocasionalmente y los espectadores que afirman no haberse visto nunca envuelto en una situación de maltrato, que son los que obtienen un promedio más bajo. Las diferencias que se observan entre ellos resultan ser significativas cuando comparamos a los espectadores puros con cada una de las restantes categorías de implicados. Es decir, 
que el grupo de víctimas, agresores, agresores-victimizados y espectadores implicados ocasionalmente, presentan medias significativamente mayores que los espectadores puros. También se aprecian diferencias al comparar las víctimas con los espectadores implicados.

Factor 2 (Desinterés académico): Los alumnos que muestran un mayor interés académico son los espectadores puros seguidos de los espectadores implicados y los agresores. Por su parte, agresores-victimizados y víctimas, por este orden, son los que mayor desinterés académico manifiestan.

Para este factor aparecen efectos estadísticamente significativos cuando comparamos las víctimas con el grupo de espectadores no implicados y con el de espectadores puros. También se aprecian diferencias al comparar a los agresores-victimizados con relación al resto de categorías (víctimas, agresores, espectadores implicados, espectadores puros), con una media más alta en aquellos. Por último, los espectadores no implicados muestran menos interés académico que los espectadores puros.

Factor 3 (Conductas disruptivas): De nuevo el grupo de víctimas, de intimidadores y de agresores victimizados presentan medias más altas que los espectadores en su conjunto. El número y la frecuencia de conductas disruptivas que exhiben estos grupos de alumnos son significativamente mayores que las que manifiestan tanto espectadores implicados como no implicados. También aparecen efectos estadísticos significativos entre el grupo de espectadores no implicados y el de espectadores puros, siendo los primeros los que obtienen medias más altas en este tipo de comportamientos desadaptados. Aunque entre las víctimas, intimidadores y agresores victimizados existen diferencias ninguna de ellas llega a ser significativa.

Factor 4 (Conductas exhibicionistas y groseras): En este factor, las víctimas y los espectadores implicados ocasionalmente obtienen mayores medias, estadísticamente significativas, que los espectadores puros. Las víctimas, a su vez, también muestran diferencias significativas, con relación a los espectadores implicados, en este tipo de conductas.

Factor 5 (Falta habilidades de comunicación con el grupo): Los agresores-victimizados, las víctimas, los agresores y los espectadores no implicados, aparecen, por este orden, como sujetos que exhiben mayor número de conductas inapropiadas a la hora de resolver un conflicto si los comparamos con los espectadores puros. Estas diferencias que se observan son todas significativas. Además puede apreciarse como los efectos estadísticos significativos que aparecen en este factor se dan también entre víctimas y espectadores no implicados y entre agresores-victimizados y los grupos de intimidadores y espectadores implicados ocasionalmente. 
Factor 6 (Conductas agresivas hacia profesores): La variable independiente "Tipología de implicados en maltrato" muestra efectos estadísticamente significativos en este factor al comparar los grupos de víctimas, agresores, agresores-victimizados y espectadores no implicados respecto al grupo de espectadores puros, que presenta el promedio más bajo. Del mismo modo, también se observa un mayor comportamiento inadecuado de este tipo en los agresores con relación a los espectadores no implicados.

Factor 7 (Conductas delictivas): Para este factor, sólo las víctimas y los espectadores implicados exhiben puntuaciones que resultan ser significativamente mayores que la de los espectadores puros.

Por último, en la puntuación total de la escala de convivencia aparecen efectos estadísticamente significativos entre el grupo de víctimas, agresores-victimizados, agresores y espectadores implicados en relación a los alumnos autoidentificados como espectadores puros. Todos ellos arrojan medias más elevadas (problemas de convivencia mayores) que estos últimos. Las víctimas y los alumnos que intimidan y son intimidados al mismo tiempo, también presenta mayores conductas desajustadas que los espectadores implicados.

Interacción entre los tipos de implicados en maltrato y el sexo respecto al CPCEA

Factor 2 (Desinterés académico): El desinterés académico parece mayor en los agresores-victimizados que en el resto de grupos, tanto en hombres como en mujeres, aunque las diferencias sólo resultan ser significativas en éstas últimas. Así, observamos medias más altas de este grupo de implicadas con relación a las víctimas del mismo sexo $(\mathrm{p}=.014)$, a las agresoras $(p=.033)$, a las espectadoras implicadas $(p=.002)$ y a las espectadoras puras $(p=.002)$. En los varones sólo se aprecia un efecto estadísticamente significativo en este factor cuando comparamos a las víctimas varones con los espectadores implicados $(\mathrm{p}=.010)$ y los espectadores puros $(\mathrm{p}=.000)$, con una media superior para las víctimas.

Por otro lado, a excepción de los agresores-victimizados, en el resto de grupos implicados en situaciones de maltrato (víctimas, agresores, espectadores implicados y espectadores no implicados) las mujeres exhiben mayor interés académico que los hombres aunque las diferencias no son todas significativas. Tan sólo han aparecido al comparar las víctimas mujeres con las víctimas varones $(\mathrm{p}=.002)$ y los espectadores implicados que son mujeres con relación a los que son hombres $(\mathrm{p}=.000)$. 
Figura 1. Efectos de la Interacción de las variables sexo y tipología de implicados con el factor de desinterés académico

Factor 2: Desinteres académico

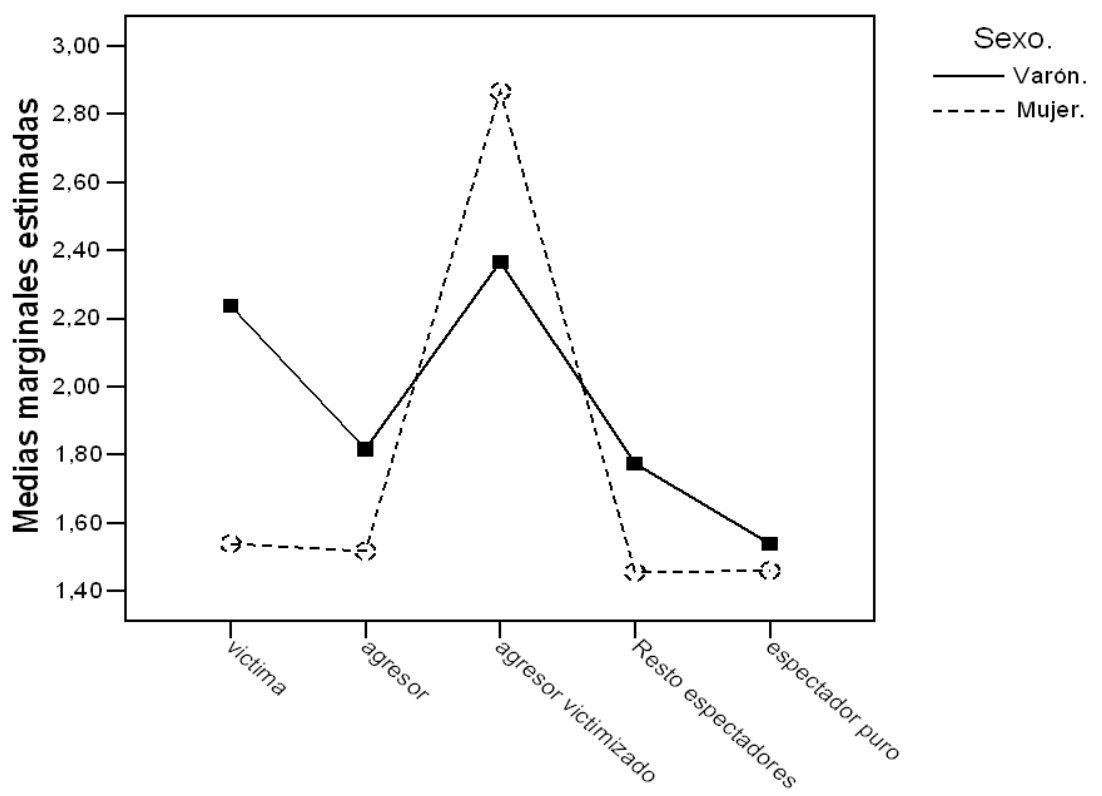

Tipología de los implicados en maltrato

Factor 5 (Falta habilidades de comunicación): Sólo en el grupo de las víctimas se aprecian diferencias significativas con respecto a este factor en función del sexo. Los chicos víctimas muestran poseer menos habilidades para resolver conflictos que las chicas - víctimas $(\mathrm{p}=.000)$

Contemplando ahora las diferencias entre las distintas tipologías según el sexo, observamos que no son las mismas en los chicos que en las chicas. Los primeros presentan medias más altas cuando actúan como víctimas que cuando son espectadores implicados $(\mathrm{p}=.000)$ y espectadores puros $(p=.000)$. En el caso de las mujeres son las agresoras-victimizadas las que manifiestan significativamente mayor falta de habilidades de comunicación al ser comparadas con las víctimas $(\mathrm{p}=.036)$, con las espectadoras implicadas $(\mathrm{p}=.018)$ y las espectadoras puras $(\mathrm{p}=.002)$.

Puntuación Total: Los mayores problemas de convivencia en general los presentan las víctimas, en el caso de los varones, y los agresores-victimizados en el caso de las hembras. Las víctimas varones muestran medias significativamente más altas que los espectadores implicados $(\mathrm{p}=.002)$ y los espectadores puros $(\mathrm{p}=.000)$. Por su parte, los espectadores implicados 
varones también presenta diferencias significativas con respecto a los espectadores puros del mismo sexo $(\mathrm{p}=.050)$. Las mujeres que están implicadas como agresoras y víctimas al mismo tiempo muestran diferencias significativas con relación a las espectadoras puras $(\mathrm{p}=.015)$.

También se observan efectos significativos al comparar a las víctimas que son chicos con las que son chicas $(\mathrm{p}=.00)$ y a los espectadores implicados varones con los que son mujeres $(\mathrm{p}=.000)$, puntuando siempre más altos los chicos.

Figura 2. Efectos de la Interacción de las variables sexo y tipología de implicados con el factor de falta de habilidades para comunicarse con el grupo

Factor5: Falta habilidades comunicación con grupo

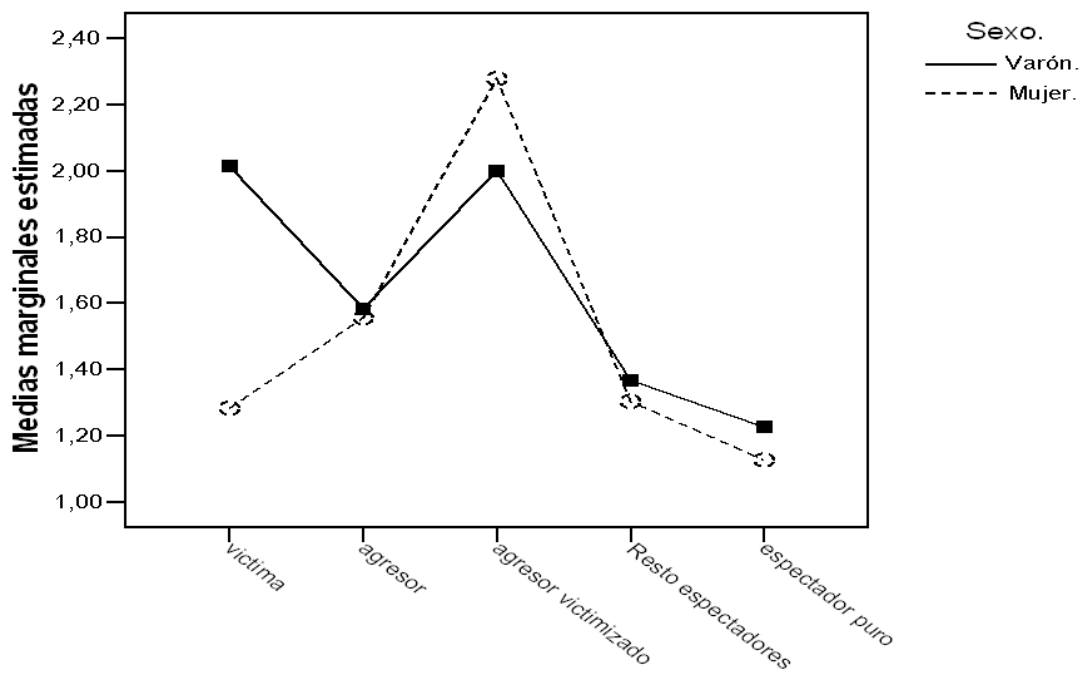

Tipología de los implicados en maltrato 
Figura 3. Efectos de la Interacción de las variables sexo y tipología de implicados con la puntuación media total del cuestionario

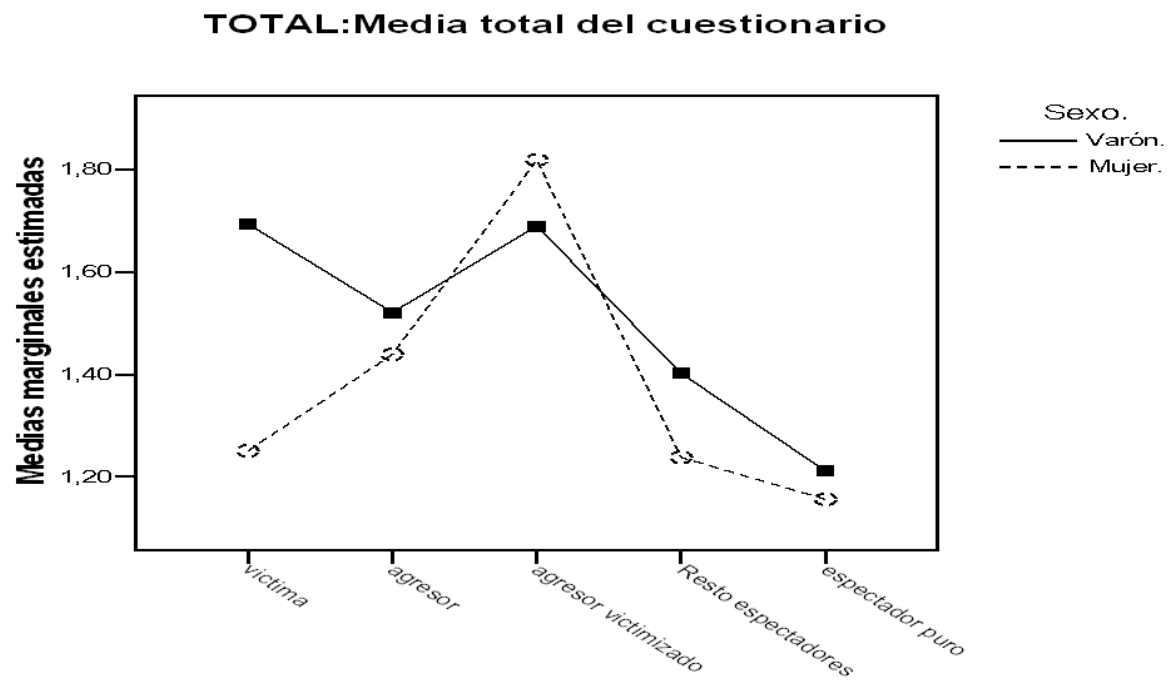

Tipología de los implicados en maltrato

\section{Discusión}

Antes de adentrarnos en la discusión sobre los problemas de convivencia de un centro y su relación con los malos tratos entre compañeros que allí se producen, vamos a trazar una panorámica de aquellos y de sus características en los centros estudiados. En primer lugar, hay que decir que el instrumento parece que se muestra eficaz para agrupar a los alumnos en función de las distintas categorías de comportamientos problemáticos que el análisis factorial ha extraído, al mismo tiempo que ayuda a dibujar la realidad de los centros estudiados con relación al conjunto de conductas desadaptadas allí presentes. En este sentido, las conductas de desinterés académico y las disruptivas aparecen como aquellas ejercidas con mayor frecuencia entre el alumnado. Les siguen las conductas agresivas hacia compañeros y la falta de habilidades para comunicarse con el grupo. Estos mismos resultados han sido obtenidos, en trabajos muy recientes, por Peralta (2004) y Sánchez (2005). En nuestro estudio aparecen otros factores, pero con menos medias que los anteriores, como son los que agrupan a conductas exhibicionistas y groseras (factor 4), a conductas agresivas hacia profesores (factor 6) y a conductas o actos delictivos (factor 7).

Por otro lado, todas estas conductas problemáticas son realizadas en mayor número por los chicos que por las chicas. El género sigue siendo, al igual que en el maltrato, una variable discriminativa con relación a los problemas de convivencia. También puede observarse cómo las conductas disruptivas, de desinterés académico y la falta de habilidades para rela- 
cionarse con el grupo son más elevadas en el colegio del extrarradio, en el que la incidencia de maltrato también es mayor. Parece ser que el maltrato entre escolares viene acompañado por el ruido de fondo que proporcionan estos otros problemas de convivencia o viceversa.

Una primera aproximación a los datos que relacionan a los escolares implicados en maltrato severo con los factores de convivencia de un centro, nos permite advertir cómo las víctimas, los intimidadores-victimizados y los intimidadores están envueltos en otros problemas de convivencia, en mayor medida que los espectadores (implicados y no implicados). Así, los agresores-victimizados son los sujetos con medias más altas en prácticamente todos los factores sobre problemas de convivencia, le siguen las víctimas y los agresores (estos alternan valores máximos en algunos factores) $\mathrm{y}$, por último, los espectadores implicados y los espectadores puros son los que menos conductas desajustadas presentan.

Algunas de estas diferencias existentes entre los protagonistas del maltrato, con relación a los problemas de convivencia detectados, son significativas y pueden proporcionarnos nuevos datos en la caracterización de los protagonistas del maltrato. Sobre todo, porque en las investigaciones revisadas durante este estudio, no hemos encontrado información a este respecto.

Si comenzamos por las víctimas, observamos que los profesores valoran, globalmente, a este grupo como el que realiza el mayor número de conductas desajustadas después de los intimidadores-victimizados y seguido muy de cerca por los intimidadores. Los problemas de convivencia que exhiben son significativamente mayores que los presentados por los espectadores implicados y no implicados.

Sorprende la percepción que los profesores tienen sobre la participación de las víctimas en la realización de conductas agresivas, si la comparamos con lo que opinan sobre los intimidadores en este mismo asunto. Supuestamente el intimidador es una persona que, con bastante frecuencia, ejerce acciones violentas contra sus compañeros. Por tanto, el promedio de agresiones tendría que ser mayor en éstos que en las víctimas y, sin embargo, es similar. Una posible explicación a este hecho puede estar en la ocultación del maltrato severo que las víctimas hacen a sus profesores, sesgando, de este modo, su percepción sobre el problema. Tal vez, lo que estén contemplando los profesores sean agresiones de frecuencia moderada, conflictos mal resueltos y no situaciones de abuso de poder, de ahí la equidad en los resultados. Puede ser también que las víctimas tengan tan asumido su papel, que las agresiones que comentan las contemplen como una reacción defensiva hacia los compañeros o como intentos de superar las situaciones de aislamiento y rechazo en las que se ven sumidas habitualmente. 
A través de los datos obtenidos en el resto de factores sobre problemas de convivencia, las víctimas se nos muestran como personas desadaptadas del entorno escolar, que entorpecen el proceso de enseñanza-aprendizaje y la dinámica de clase, que expresan poco interés por el trabajo del aula, que molestan frecuentemente a compañeros y profesores, que incumplen las normas básicas de funcionamiento del centro y que manifiestan un déficit claro en habilidades para desenvolverse en grupo. De todas estas conductas, las de desinterés académico y falta de habilidades para relacionarse con sus compañeros son las que están por encima de la media de las del resto de implicados en maltrato, a excepción de los intimidadores-victimizados.

Hay variables como el sexo que ayudan a matizar estos resultados Las chicas víctimas mantienen un buen nivel de interés académico, similar al del resto de alumnos implicados, mientras que los alumnos varones que son víctimas presentan un desinterés elevado, siendo las diferencias con aquellas significativas. Con relación a la falta de habilidades para relacionarse en grupo, ocurre algo parecido: las chicas víctimas obtienen medias muy bajas en este factor y los chicos muy altas, siendo la diferencia significativa. No parece pues que, en el caso de las chicas, el desinterés académico y la falta de habilidades para la comunicación en grupo sean una consecuencia del proceso de victimización, ni un factor causal. No podemos afirmar lo mismo en el caso de los chicos. Para las víctimas que son mujeres hay que buscar en otros factores de convivencia el efecto o la causa de la victimización, por ejemplo, en la disruptividad o en las conductas agresivas hacia sus compañeros donde los valores que aparecen están muy por encima de la media que presentan los escolares no implicados en maltrato.

Si nos detenemos ahora en los intimidadores, observamos como éstos se acercan basstante al nivel de conflictividad que mantienen las víctimas, aunque con diferencias muy características.

Las conductas disruptivas y los comportamientos agresivos dirigidos hacia los profesores son las dos categorías de problemas de convivencia en las que los intimidadores destacan por encima de las víctimas, aunque no de manera significativa. Con quienes sí mantienen diferencias sustantivas es con los espectadores implicados y los espectadores puros.

Constituyen, como podemos ver, comportamientos problemáticos que deterioran la convivencia en los centros y la marcha normal de clase mediante un ataque directo o indirecto a sus profesores. Puede que estos retos a la figura del profesor estén relacionados con el estatus sociométrico que ellos han adquirido dentro del grupo (Estell, Cairns, Farmer y Cairns, 2002; Estell, Farmer, Pearl, Van Acker y Rodkin, 2003; Mora-Merchán, 2000; Olweus, 1989; Ramírez, 2006; Rodkins, Farmer, Pearl, y Van Acker, 2000; Whitney, Nabuzoka y Smith, 
1992). Necesitan, quizás, demostrar a los demás que son capaces de cuestionar la autoridad de quien la ostenta dentro del aula, enfrentándose a él. Probablemente busquen reforzar y fortalecer la posición de poder que ocupan con respecto a sus compañeros.

Por otro lado, las conductas agresivas dirigidas a sus compañeros, el desinterés académico, la falta de habilidades para vivir en grupo, junto a la realización de actos delictivos y de conductas exhibicionistas, son acciones menos habituales o de igual frecuencia en los agresores que en las víctimas y siempre mayores que en los espectadores de ambos tipos.

Si relacionamos estos resultados obtenidos por los intimidadores en los problemas de convivencia con la variable sexo, tal y como hicimos en el caso de las víctimas, observamos que los intimidadores, ya sean chicos o chicas manifiestan el mismo nivel de conductas disruptivas, de desinterés académico y de falta de habilidades para relacionarse con el grupo.

Como hemos podido ver, intimidadores y víctimas parecen evolucionar de manera distinta, con relación a los problemas de convivencia, cuando inciden en ellos variables como el sexo.

Los espectadores implicados y los espectadores puros presentan menos problemas de convivencia en todos los factores que el resto de escolares que participan en situaciones de maltrato. Y entre ambos tipos de espectadores, los no implicados son los que apenas exhiben comportamientos inapropiados en las aulas y en el centro. Por tanto, la hipótesis que planteábamos sobre el vínculo que podía existir entre la frecuencia de conductas problemáticas para la convivencia y el tipo de implicación en situaciones de maltrato es congruente con la evidencia empírica. Así, los intimidadores-victimizados, que son los que con más intensidad viven el maltrato (Collell y Escudé, 2004; Duncan, 1999; Smith, 2004; Schwartz, Dodge, Petit y Bates, 2000; Wolke, Woods, Bloomfield y Karstadt, 2000) manifiestan más conductas desajustadas; les siguen las víctimas y los agresores (protagonistas, también directos, de estas situaciones) y los que resultan ser menos problemáticos son los espectadores implicados y los espectadores puros, sujetos que se ven menos envueltos en escenarios de abuso.

Los espectadores implicados presentan un mayor número de conductas problemas en todas las categorías estudiadas si los relacionamos con los espectadores puros, siendo estas diferencias significativas. Este incremento que se observa en los espectadores involucrados ocasionalmente es equivalente, en todas las conductas, al que presentan las víctimas pero con menor promedio. Es interesante contemplar como estos implicados ocasionales, aparecen como un grupo intermedio, de transición, entre los espectadores puros y las víctimas. 
En consonancia con lo que acabamos de decir, al introducir la variable sexo se repite en gran medida lo que ocurría con las víctimas pero con puntuaciones menos elevadas: los chicos que son espectadores implicados expresan mayor desinterés académico que las chicas, de forma significativa; cosa que no ocurre con los espectadores puros. Las conductas exhibicionistas y groseras y la falta de habilidades para desenvolverse en el grupo siguen el mismo patrón. Las advertencias sobre la necesidad de considerar la intimidación como una serie continua en la que se contemplen comportamientos agresivos de intensidades diferentes, ya lo hicieron autores como Espelage, Bosworth y Simon (2001) o Hoover, Oliver y Thompson (1993) quienes apostaban porque en la investigación tomara cada vez más cuerpo este tipo de comportamientos que por ser muy comunes y aparentemente de bajo nivel, pudieran no ser reconocidos como graves y necesitados de intervención (Craig, Henderson y Murphy, 2000).

Para concluir, manifestar que los intimidadores-victimizados son catalogados como los sujetos que se ven envueltos en un mayor número y variedad de conductas problemáticas para la convivencia en comparación con el resto de sus compañeros. No obstante, los resultados relativos a este grupo de escolares deberían ser revisados en otras investigaciones en el que el número de sujetos fuera mayor. 


\section{Referencias}

Almeida, A. (1999). Portugal. En P.K. Smith; Y. Morita, J. Junger-Tas, D. Olweus, R. Catalano y P. Slee (Eds.), The nature of School Bullying : A cross-national perspectiva, (pp. 174-187). Londres: Routledge.

Benítez, J.L. (2003). Diseño, implementación y evaluación de un programa de intervención contra los malos tratos entre iguales en contextos escolares. Tesis doctoral. Universidad de Granada.

Calvo. A. R. (2003). Problemas de convivencia en los centros educativos. Análisis e intervención. Madrid: EOS

Collell, J. y Escudé, C. (2004). Rol de les emocions en els processos de maltractament entre alumnes. Ambits de Psicopedagogía, 12, 21-26.

Craig, W.M., Henderson, K. y Murphy, J.G. (2000). Prospective teachers' attitudes toward bullying and victimization. School Psychology International, 21, 5-21.

Duncan, R. D. (1999). Maltreatment by parents and peers: The relationship between child abuse, bully victimization, and psychological distress. Child Maltreatment, 4, 45-55.

Espelage, D.L., Bosworth, K. y Simon, T.R. (2001). Short-term stability and prospective correlates of bullying in middle-school students: An examination of potential demographic, psychosocial, and environmental influences. Violence and Victims, 16, 411426

Estell, D.B., Cairns, R.B., Farmer, T.W. y Cairns, B.D. (2002). Aggression in Inner-City Early Elementary Classrooms:Individual and Peer Group Configurations. MerrillPalmer Quarterlym 48, 52-76.

Estell, D., Farmer, T., Pearl, R., Van Acker, R. y Rodkin, P. (2003). Heterogeneity in the Relationship Between Popularity and Aggression: Individual, Group and Clasroom influences. New directions for child and adolescent development, 101, 75-85.

Etxeberria, J., Joaristi, L. y Lizasoain, L. (1990). Programación y análisis estadísticos básicos con SPSS/PC+. Madrid: Paraninfo.

Hoover, J.H., Oliver, R. y Thompson, K. (1993). Perceived victimization by school bullies: New research and future directions. Journal of Humanistic Education and Development, 32, 76-84.

Ianni, N.D. (2003). La convivencia escolar: una tarea necesaria, posible y compleja. Organización de Estados Iberoamericanos. Monografías $N^{o} 2$ (agosto-septiembre), Consultada el 3 de noviembre de 2003 en: 
http://www. campus-oei.org/valores/monografias02/reflexion02.htm

Ianni, N.D. y Pérez, E. (1998). La convivencia en la escuela: un hecho, una construcción. Buenos Aires: Paidós.

Kruskal, W.H. y Wallis, W.A. (1952). Use of ranks in one-criteriom variance analysis. J. Amer. Statis. Assn. 47, 583-621.

Long, J. D., y Pellegrini, A. D. (2003). Studying change in dominance and bullying with linear mixed models. School Psychology Review, 32, 401-417.

Mora-Merchán, J.A. (2000). El fenómeno bullying en las escuelas de Sevilla. Tesis doctoral. Universidad de Sevilla.

Morollón, M. (2001). Una panorámica sobre la convivencia en los centros. Cuadernos de Pedagogía, 304, 42-54.

Olweus, D. (1989). The Olweus Bully/Victim questionnaire (Mimeo). HEMIL,-senteret, Universitetet I Bergen, Norway. Bergen, Norway: University of Bergen.

Olweus, D. (1993). Bullying at school: What we know and what we can do. Oxford, UK.: Blackwell.

Ortega, R. (2002). El maltrato entre iguales, uno de los problemas de violencia escolar, no el único. Federación de Enseñanza de CCOO. Consultado el día 23 de marzo de 2003 en http://wwwfe.ccoo.es/poleduc/foro_convivencia/foro.htm

Peralta, F. J. (2004). Estudio de los problemas de convivencia escolar en estudidantes de enseñanza secundaria. Tesis doctoral. Universidad de Málaga.

Pereira, J. (2002). Violencia y Escuela. Consultado el 18 de noviembre de 2002 en http://www.redeseducacion.net/violen002.htm

Ramírez, S. (2006). El maltrato entre escolares y otras conductas-problemas para la convivencia: Un estudio desde el contexto del grupo-clase. Tesis Doctoral. Universidad de Granada.

Rodkins, P. C., Farmer, T. W., Pearl, R., y Van Acker, R. (2000). Heterogeneity of popular boys: Antisocial and prosocial configurations. Developmental Psychology, 36, 14-24.

Rodkins, P.C. y Hodges, E.V.E. (2003). Bullies and victims in the peer ecology: Four question for school service providers and social development research. School Psychology Review, 32, 384-400

Salmivalli, C., Lagerspetz; K. Bjoerkqvist, K. y Oesterman, K y Kaukianinen, A. (1996). Bullying as a group process: Participant roles and their relations to social status within the group. Aggressive Behavior, 22(1), 1-15. 
Sánchez, M. D. (2005). Creencias sobre razonamiento moral y conflictos en adolescentes con problemas de adaptación escolar. Tesis Doctoral. Universidad de Málaga.

Schwartz, D., Dodge, K. A., Pettit, G. S., y Bates, J. E. (2000). Friendship as a moderating factor in the pathway between early harsh home environment and later victimisation in the peer group. Developmental Psychology, 36, 646-662.

Smith, P.K. (2004). Bullying: Recent Developments. Child and Adolescent Mental Health 9(3), 98-103.

Solberg, M. y Olweus, D. (2003). Prevalence estimation of school bullying with the Olweus bully/victim questionnaire. Aggressive Behavior, 29, 239-268.

Swearer, S. M., Song, S. Y., Cary, P. T., Eagle, J. W., y Mickelson, W. T. (2001). Psychosocial correlates in bullying and victimization: The relationship between depression, anxiety, and bully/viitim status. In R. A. Geffener, M. Lorieng y C. Young (Eds.), Bullying behaviour: Current issues, research, and interventions (pp. 95-121). Binghamton, NY: Haworth Maltreatment y Trauma Press.

Tejedor, F.J. (1984). Análisis de varianza aplicado a la investigación en Pedagogía y Psicología. Madrid: Anaya.

Torrego, J.C. y Moreno, J.M. (2003). Convivencia y disciplina en la escuela. El aprendizaje de la democracia. Madrid: Alianza.

Whitney, I., Nabuzoka, N. y Smith, P. K. (1992). Bullying in schools: Mainstream and special needs. Support for Learning, 7, 3-7.

Wintney, I. y Smith, P.K. (1993). A survey of the nature and the extent of bullying in junior, middlle and secondary schools. Educational research, 35, 3-25.

Wolke, D., Woods, S., Bloomfield, L., y Karstadt, L. (2000). The association between direct and relational bullying and behavior problems among primary school children. Journal of Child Psychology and Psychiatry, 8, 989-1002. 
El maltrato entre escolares y otras conductas-problemas para la convivencia

[Página en blanco por razones de paginación] 